\title{
History and Facts about Black Holes
}

\section{Devandra Khatiwoda}

Prithwi Narayan Campus, Pokhara

The term black hole was coined in 1969 by the American scientist John Wheeler. Historian also believes that the oldest mention of black hole was found in the books of British history of India. The feud between the East India Company and the ruler of Bengal named Nawab Siraj-Ud-daula in 1757 lead to a macabre incident famously known as "Black hole of kalkata." Hundred forty six warrior of East India Company who survived after feud won by Nawab were ordered to imprison by the furious Nawab in a prison cell, 16 feet (about 5 meter) by 14 feet (about $4 \mathrm{~m}$ ) which only had capacity for imprisonment of 3 or 4 soldiers. During 10 hours of imprisonment 123 prisoners died. This region of prison was as if a physical black hole. Also, according to Hindu religion book to kill a danav named Raktabij who had got a boon from Brahma to have thousands replica of himself from a single dropped out blood Goddess Kali had to create a region in her mouth which would feed crores of that replicated danav. This region in her mouth can also be compared with a physical black hole whose mass concentration generates a gravitational attraction so strong that not even a single ray of light can escape from its surface. Who first thought about this bizarre object?

The French Mathematician, Laplace conceived of notion of such an object in the year 1799. Although he didn't mention the term black hole but idea was concerned about the property of escape speed exceeding the speed of light. For any object to escape from earth's surface its speed must be 11.2 $\mathrm{km} / \mathrm{hr}$ called escape speed. If the speed would be some what less it would not escape the gravitational attraction of earth. Comparing the object as particle of light (photons) escaping the gravitational attraction, a high gravitation if could be created such that it would not let the photon to escape, a region according to Laplace is formed which looks completely black from outside region.
The critical value for the radius of smaller object not to let light escape as to become a Laplace black hole was given as,

$$
R_{S}=\frac{2 \mathrm{GM}}{C^{2}}
$$

Where $\quad \mathrm{R}_{\mathrm{s}}=$ Radius of Laplace black hole, $\mathrm{C}=$ Velocity of light, $\mathrm{G}=$ Universal gravitational constant $=6.67 \times 10^{\wedge}(-11) \mathrm{Nm}^{2} / \mathrm{Kg}^{2}$. For earth this radius is of the order of $5 \mathrm{~mm}$ and for sun it is about $3 \mathrm{~km}$.

However, Laplace was not only the physicist to talk about black hole. A Cambridge physicist John Michell wrote a paper in the philosophical translation of the Royal Society of London (vol. 84, pp35). In it, he pointed out that the star which was sufficiently massive and compact would not let even a single ray of light to escape from it, if had a high gravitational attraction. The star thus becomes invisible, even though their gravitational attraction may be felt. Such objects are what we now call black holes, because that is what they are- black voids in space.

To understand how a black hole might be formed we must first understand the life cycle of a star. The star remains in its stable configuration due to inward and outward force balancing each other like an inflated balloon which is balanced between the pressure and the tension in rubber which is trying to make balloon smaller. In case of star the inward force is created due to gravitational attraction and the outward force due to high pressure created by nuclear fusion reaction (reaction between hydrogen gases forming helium). When the hydrogen is completely converted to helium in center core of star there is no more production of heat creating an outward pressure. As a result there is only an inward gravitational attraction. Due to this the star contracts 
continuously and temperature of star core increases tremendously causing the expansion of outer layer. Due to expansion, the temperature of outer layer starts falling. Finally a stage is reached when a star becomes large in size and red in color called red giant. A star may remain as a red giant for several years. At the end stage a violent explosion called Nova or Supernova occurs which throws a large portion of star into the space. This is called death of star.

When the star tends to reach death its initial mass at the time plays an important role to decide death. The core of star may end up in three different ways: White Dwarf, Neutron star and Black hole.

To understand how these three are formed we must have some knowledge about "Chandrasekhar limit." Subramanian Chandrasekhar, an Indian astrophysicist worked on how big a star could be to still support its own gravity after it has used all its fuel, by the implication of "degeneracy pressure" exerted in a closed packed system of particles. Quantum physics describes the behavior of particles in very small scale of atom and nuclei. When identical particles are packed in a small volume they are endowed by a pressure called "degeneracy pressure" which has tendency to resist closer packing and therefore help to maintain the stars equilibrium against its self gravity. This "degeneracy pressure" can be explained on basis of Pauli's exclusion principle as the particles come close to each other. According to Pauli's exclusion principle the matter particles can not have same position and velocities. So they move with different velocity which makes the matter particles to move away from each other as a result star tends to expand. A star can therefore maintain at constant rate by the balance between the inward pull and repulsion due to Pauli's exclusion principle.

Chandrasekhar however found that there is a limit to the mass that it can be held in equilibrium through the degeneracy pressure arising from closed packing of electron. This limit is known as Chandrasekhar limits and is 1.4 solar mass. Thus simply understanding that if mass of remnant (mass remaining after explosion of red giant) is small than Chandrasekhar limit it would endowed by a degeneracy pressure and star may either end up as white dwarf or neutron star but if the mass of remnant exceed Chandrasekhar limit the "degeneracy pressure" will not be capable of holding the star's self gravity and a high gravitational collapse would result formation of black hole.

White dwarf: If the initial mass of star is less than two times mass of sun i.e. remnant has mass less than Chandrasekhar limit star ends up as white dwarf and its size condenses to size of earth. Its color changes from white to yellow and then to red and finally becomes black. White dwarf has radius of about few thousand miles and density of hundred of tons per cubic inch. White dwarf is supported by an exclusion principle between the electrons in matter. One of the first observed white dwarf is star that is orbiting around Sirius the brightest star at night sky.

Neutron star: If the mass of star is between two to five times mass of sun or the remnant has mass less than Chandrasekhar limit, the red giant becomes a neutron star. When stars mass is greater than two times mass of sun the contraction of star is thought to continue until a high density is reached. At this stage electron collides with proton producing neutron star. This star does not emit light but sends radio waves in form of pulses, so also called pulsar. Neutron star is supported by exclusion principle (caused by degeneracy pressure of closed packed neutron about $10^{44}$ particles per cubic meter) between neutron and proton rather than electron. Neutron star has diameter about $10-24 \mathrm{~km}$ and density $10^{17} \mathrm{~kg} / \mathrm{m}^{3}$ with intense magnetic field of order $10^{8}$ Tesla.

Black holes: If the initial mass of star was five times the mass of sun or the remnant has mass exceeding Chandrasekhar limit, the remnant can not survive as a white dwarf or neutron star. Its pressure will be inadequate to withstand the crushing force of its own gravity. The star continues to contract. This is the situation of gravitational collapse. From the initial gentle rate of contraction, the star progresses to a catastrophic implosion when its self-gravity becomes so strong that nothing, even a ray of light, can prevent from it resulting the information of 
black hole. In order to become a black hole for our sun it must contract to a radius of about $3 \mathrm{~km}$. The black hole can only be felt by two ways as by its very definition it does not emit any light.

1) By the field on its neighboring objects in the sky:

Astronomers have observed a number of system in which two stars orbit around each other, attracted towards each other by gravity. They also observed system in which there is only one visible star moving towards black hole.
2) By the direction of $X$-rays given by black hole. The matter blown off from the surface of visible star when falls toward unseen company, it develops spiral motion i.e. material swirls off and becomes very hot emitting $\mathrm{X}$-ray. From the observed motion of the visible star, one can determine lowest possible mass of sun. So according to Chandrasekhar's result this mass is too large for an unseen object to be a white dwarf or a neutron star. So must be a black hole. 